\title{
Brain cortical and subcortical morphology in adolescents with depression and a history of suicide attempt
}

\author{
Anthony J. Gifuni, MD, MSc; M. Mallar Chakravarty, PhD; Martin Lepage, PhD; \\ Tiffany C. Ho, PhD; Marie-Claude Geoffroy, PhD; Eric Lacourse, PhD; Ian H. Gotlib, PhD; \\ Gustavo Turecki, MD, PhD; Johanne Renaud, MD, MSc*; Fabrice Jollant, MD, PhD*
}

\begin{abstract}
Background: Suicidal behaviours are a major source of mortality and morbidity among adolescents. Given the maturational changes that occur in cortical and subcortical structures during adolescence, we tested whether atypical brain structural measurements were associated with a history of suicide attempt. Methods: We assessed 3 groups of adolescents $(n=92 ; 79 \%$ female, mean age 15.9 years, range 11.6-18.1 years): patients with a depressive disorder and a history of suicide attempt $(n=28)$; patient controls, who had a depressive disorder but no history of suicide attempt $(n=34)$; and healthy controls $(n=30)$. We derived regional cortical thickness and surface area, and subcortical volumes, from $T_{1}$-weighted anatomic MRI scans acquired at $3 \mathrm{~T}$. Results: We found significant group differences in surface area in the prefrontal, temporal and parietal regions, as well as in the volume of several subcortical nuclei $\left(p_{\mathrm{FDR}} \leq 0.05\right)$, but not in cortical thickness. Post hoc analyses indicated that morphological alterations primarily differentiated patients with a history of suicide attempt from healthy controls, but not from patient controls. However, patients with a history of suicide attempt exhibited positive correlations between age and cortical thickness in the temporal cortices and right insula, and between age and right putamen volume (i.e., thicker regional cortex and larger subcortical volumes with age). These correlations were negative in both patient controls and healthy controls (i.e., thinner regional cortex and smaller subcortical volumes). Limitations: Sample sizes, cross-sectional findings and psychiatric heterogeneity were limitations of this study. Conclusion: Macroscopic structural differences in several brain regions differentiated adolescents with a history of suicide attempt from healthy controls, but not from patient controls. However, adolescents with a history of suicide attempt may present with atypical maturation of specific cortical and subcortical regions that might contribute to the risk of suicidal behaviour.
\end{abstract}

\section{Introduction}

Suicide is the second leading cause of death in adolescents. ${ }^{1}$ A recent survey of college students showed that lifetime prevalence of suicidal ideation, plans and attempts were $32.7 \%, 17.5 \%$ and $4.3 \%$, respectively, and that the onset of suicidal thoughts and behaviours occurred before age 16 years in $75 \%$ of cases. ${ }^{2}$ Examining suicidality in adolescence is critical, given that this period is associated with the sharpest increase in suicidal thoughts and behaviours. ${ }^{3}$ Further, suicidality in adolescence is associated with poorer adult outcomes in terms of future suicide risk and increased risk of psychopathology and functional impairment. ${ }^{4}$

There are significant challenges in conducting suicide risk assessment. For instance, self-report measures of suicidality are limited by reporting biases (e.g., concealing suicidality to avoid anticipated negative consequences) and high temporal variability (i.e., moment-to-moment fluctuations in selfreported suicidal ideation ${ }^{5}$ ). Moreover, classical clinical and demographic risk factors are of limited predictive value. ${ }^{6}$ Theoretical models of suicidal behaviours increasingly emphasize the critical role of neurobiological factors. ${ }^{7}$ Accordingly, new approaches are needed to identify neural markers associated with the emergence of suicidal behaviours during adolescence. Furthermore, identifying adolescent biomarkers could provide malleable therapeutic targets during early intervention and expand our developmental understanding of adult suicidality.

Advances in neuroimaging research suggest that measures of brain function and structure may represent relevant avenues of investigation. ${ }^{8,9}$ More recent structural studies ${ }^{8,9}$ have used surface-based morphometry, which decomposes

Correspondence to: F. Jollant, Clinique des Maladies Mentales et de l'Encéphale (CMME), Hôpital Sainte-Anne, 100 rue de la santé, 75674 Paris, Cedex 14, France; fabrice.jollant@ parisdescartes.fr

Submitted Oct. 21, 2020; Revised Dec. 23, 2020; Accepted Jan. 14, 2021

${ }^{*}$ Co-senior authors.

DOI: $10.1503 / j p n .200198$ 
cortical volume into its 2 primary components: cortical surface area and thickness. These components have distinct evolutionary origins ${ }^{10}$ and are regulated by different genetic and cellular processes. ${ }^{11,12}$ A recent meta-analysis with 18925 adult participants (6448 with depression, of whom 694 had a history of suicide attempt) indicated that on average, people with depression who had attempted suicide had a lower left inferior parietal lobe surface area than people with depression who had not attempted suicide, ${ }^{13}$ but the 2 groups showed no differences in cortical thickness. In addition to identifying differences in cortical surface area, the same meta-analysis found that smaller volumes of the bilateral thalami and right pallidum characterized people who had attempted suicide compared to clinical controls. These structural variations were located in key integrative regions, encoding reward-oriented behaviour, social cognition, affect regulation or higher-order cognition. ${ }^{14,15}$ Hence, disruptions in cortical and subcortical networks might mediate adult suicidal behaviours through complex emotional and behavioural mechanisms. ${ }^{16}$ The origin of macroscopic neural alterations associated with adult suicidal behaviours remains unclear, further highlighting the need to examine neuroimaging correlates in younger cohorts.

Several neuroimaging studies have reported an association between cortical morphometric alterations and a history of suicidal behaviours in adolescents, although not all studies have found statistically significant differences between patients with and without a history of suicide attempt. For instance, adolescents (age 12-17 years) with major depressive disorder (MDD) and a history of suicide attempt have, on average, smaller grey matter volume in the right superior temporal gyrus than healthy controls, but not adolescents with MDD and no history of suicide attempt. ${ }^{17}$ Smaller volumes in the superior temporal gyrus were also found in a group of adolescents with treatment-resistant depression who had attempted suicide (age 12-21 years) compared to a group of healthy controls. ${ }^{18}$ In slightly older patients (age 1425 years) with a mood disorder (MDD or bipolar disorder), Fan and colleagues ${ }^{19}$ found that a history of suicide attempt was associated with smaller volumes in the ventromedial and dorsolateral prefrontal cortices. Further, youth with MDD and a history of suicide attempt were characterized by smaller volumes in the dorsomedial prefrontal region compared to controls with a mood disorder but without a history of suicide attempt. Researchers also found that a history of suicide attempt in youth (age 14-25 years) with bipolar disorder was associated with smaller left lateral orbitofrontal volume than healthy controls but not patient controls. ${ }^{20}$ One longitudinal investigation of suicide risk in the same sample also found that smaller ventral anterior prefrontal volume at baseline predicted suicide attempts during a 3-year follow-up period, although the authors found no change in grey matter volume. ${ }^{21}$ Hence, suicidal behaviours in youth are associated with structural anomalies in prefrontal and temporal regions, which might be linked to neurobehavioural alterations associated with adolescent suicide risk..$^{22}$ Indeed, adolescent suicide risk has been mainly associated with cognitive control and decision-making deficits, ${ }^{22}$ but the exact mapping of these def- icits to structural alterations remains unknown. Investigating surface area or cortical thickness as biomarkers of adolescent suicide risk represents an important step in elucidating the neurocognitive mechanisms that predispose youth to suicidal behaviours.

There is less evidence linking morphological characteristics in subcortical structure with adolescent suicidal behaviours. One study found an association between striatal grey matter volume and adolescent suicidality, but only when examining implicit associations between the self and suiciderelated content. ${ }^{23}$ Cross-sectional studies have also suggested an association between hippocampal volumes and history of suicide attempt in youth with bipolar disorder, but not youth with MDD. ${ }^{19,21}$ Thus, additional studies are warranted to clarify the potential role of subcortical volumes as suicide biomarkers in adolescence. It is particularly important to examine subcortical volumes in conjunction with cortical structures, because the interactions of these structures are thought to be involved not only in the physiopathology of suicide, ${ }^{9}$ but also more generally in the emergence of adolescent problematic behaviour. ${ }^{24}$

Studies have mainly examined global group differences in brain measures, but adolescence is characterized by substantial neurodevelopmental change over this relatively short period. Recent neuroimaging analyses combining several samples of adolescents suggest that cortical thinning is the primary factor that drives nonlinear decreases in cortical volume. ${ }^{25}$ Whereas some cortical regions show a decrease in thickness (e.g., lateral prefrontal cortex), others show either an increase or decrease (e.g., anterior cingulate). ${ }^{26}$ Furthermore, changes in surface area - partly independent of changes in cortical thickness - also occur in a regionally specific manner. ${ }^{25}$ Importantly, subcortical structures also continue to undergo maturational changes during adolescence. ${ }^{27}$ The relationship between these maturational changes and the emergence of suicidal behaviours during adolescence remains unknown. Additional studies should examine whether age-related factors affect the association between brain structural variation and suicidal behaviours in adolescence.

In summary, few studies have examined the association between brain morphological features and suicidal behaviours in adolescents. Those that have explored this topic have reported inconsistent findings and have not generally accounted for developmental changes and individual variability, focusing instead on mean group differences. In the current study, we aimed at addressing these important gaps in the literature. We examined a large clinical sample of wellcharacterized adolescents with depression, with and without a history of suicide attempt, from a specialized psychiatric outpatient clinic in which adolescents with complex cases of depression receive mental health care. We recruited only patients with depressive disorders to limit clinical heterogeneity. First, as previously done, we sought to replicate cross-sectional findings relating cortical morphology to adolescent suicidal behaviours, using a well-validated analytic pipeline that examined both cortical thickness and surface area. We also examined group differences in subcortical volumes (striatum, amygdala, hippocampus, 
thalamus). Based on previous work conducted in adolescents, we hypothesized that adolescent suicidal behaviours would be associated with anomalies in cortical surface and thickness located mainly in the frontal, insular or temporal cortex. In line with neuropsychiatric and neurodevelopmental models, ${ }^{9,25}$ we hypothesized that cortical regions associated with suicidal behaviour would be characterized by reduced surface area and increased cortical thickness. For subcortical regions, we hypothesized that a history of suicidal behaviour would be associated with relative atrophy in subcortical limbic regions (striatum, amygdala or hippocampus). We expected that most structural differences associated with adolescent suicidal behaviours would also be found in adolescents who had depression but no history of suicide attempt, given the overlaps in psychiatric symptomatology in this group. Second, we examined the interaction of age and morphological measures in each group, which we posited would be significant if atypical maturational trajectories underlay suicide risk. Because cortical thickness tends to decline linearly in the age range of our sample, we expected that an abnormal neurodevelopmental trajectory associated with suicidal behaviours would present as either attenuation or reversal of the linear trends found in adolescents without a history of suicide attempt.

\section{Methods}

\section{Participants}

We recruited a total of 104 adolescents, aged 11-18 years, and categorized them into 1 of 3 groups: adolescents with a depressive disorder and a history of at least 1 suicide attempt ( $n=30 ; 26$ female); adolescents with a depressive disorder and no history of suicide attempt (patient controls; $n=38$; 34 female); and adolescents with no history of a psychiatric disorder or suicide attempt (healthy controls; $n=36$; 26 female). Exclusion criteria included neurological disorders (e.g., epilepsy, brain tumour), traumatic brain injury (> 1 minute loss of consciousness, neuroimaging anomaly, persistent post-concussive symptoms), autism spectrum disorder, bipolar disorder, psychotic disorder, IQ less than 70, pregnancy and any contraindication for MRI imaging. Given the evidence that some phenotypes associated with suicide attempts are heritable, including neuroimaging markers, ${ }^{28,29}$ a family history of suicide attempt was an additional exclusion criterion for the healthy control group (but not for the patient control group). Three participants (1 patient control and 2 healthy controls) did not complete the MRI assessment because of claustrophobia or refusal. Nine participants were excluded from analyses because of poor scan quality. Therefore, analyses included 28 adolescents with a depressive disorder and a history of suicide attempt, 34 patient controls and 30 healthy controls.

Recruitment took place from September 2012 to January 2019, primarily at the Depressive Disorders Clinic for adolescents at the Douglas Institute in Montréal, Quebec, Canada; additional participants were recruited from existing studies or referred from child psychiatrists in affiliated community clinics. Access to these specialized psychiatry services were limited to pediatric populations, which led to the pragmatic decision to include only participants aged 18 years or younger. Healthy controls were recruited from the community through advertisements posted in schools, local clinics and hospitals, youth centres and groups of parents on social media. Participants were initially screened via phone interview by a trained research assistant. All participants were compensated financially (\$50 for the complete assessment), and consent was obtained from adolescents and a least 1 of their parents or legal guardians. The research protocol was approved by the Douglas Institute Research Ethics Board.

\section{Assessments}

Consistent with the recommendations for a consistent nomenclature of suicidal events, ${ }^{30,31}$ a suicide attempt was defined as a self-injurious behaviour carried out with some intent to die; therefore, aborted and interrupted suicide attempts (attempts halted by the participant or another person before being carried out) and nonsuicidal self-injuries were not included. Similarly, patients with exclusively nonsuicidal self-injuries were excluded from the group of patients with a history of suicide attempt. We characterized suicidal behaviours using the Suicide History Questionnaire (in-house questionnaire), cross-validated with notes from the patient's medical files (reviewed by A.J.G.) and through a clinical interview with a child and adolescent psychiatrist (J.R.). All participants were interviewed using the Kiddie Schedule for Affective Disorders and Schizophrenia - Present and Lifetime. ${ }^{32,33}$ This assessment characterized the type of depressive disorder (MDD, depressive disorder not otherwise specified or dysthymia), along with psychiatric comorbidities. The clinical interview also assessed whether participants had a lifetime history of nonsuicidal self-injury. We also assessed current depressive symptoms using the selfreport Beck Depression Inventory-II. ${ }^{34}$ We measured participants' intelligence (IQ) using the Wechsler Intelligence Scale for Children, 4 th edition, ${ }^{35}$ in participants younger than 17 years and the Wechsler Adult Intelligence Scale, 4th edition, ${ }^{36}$ in participants 17 years and older.

\section{Imaging procedure}

We acquired structural $T_{1}$-weighted MRI brain scans at the Douglas Brain Imaging Centre using a Siemens Magnetom Trio (Tim System 3 T, MR B17) MRI scanner with a 12-channel head coil. We used a magnetization-prepared rapid gradient echo sequence with the following parameters: repetition time $2300 \mathrm{~ms}$; echo time $2.98 \mathrm{~ms}$; flip angle $=9^{\circ}$; field of view $256 \mathrm{~mm}$, voxel size $1 \mathrm{~mm}^{3}$ isotropic; acquisition time $9.25 \mathrm{~min}$.

\section{MRI preprocessing and data extraction}

Structural MRIs underwent reconstruction using the fully automated and well validated segmentation software FreeSurfer v5.3, ${ }^{37}$ which is open-source and freely available 
(http:/ / surfer.nmr.mgh.harvard.edu/). Surface-based morphometry ${ }^{38}$ yielded estimations of cortical surface (area at pial surface) and thickness (distance between the pia matter and the underlying grey-white matter interface). We also visually inspected brain segmentations of the cortex and subcortical grey matter regions, focusing on overall image quality, including motion artifacts and the overall accuracy of whole-brain volumetric segmentations and the reconstructed surfaces. Scans that were of poor quality because of widespread segmentation errors were excluded from the analysis. No manual editing was performed.

We extracted the average cortical thickness and surface area of the 34 bilateral cortical regions defined by the Desikan-Killiany surface atlas, ${ }^{39}$ as well as the volume of 7 bilateral subcortical structures (nucleus accumbens, caudate nucleus, putamen, pallidum, thalamus, amygdala and hippocampus).

\section{Statistical analyses}

We performed all statistical analyses in R v3.6.0, ${ }^{40}$ implemented in Rstudio v1.1.383. ${ }^{41}$ We first examined group differences using univariate general linear models (1-way analyses of covariance), with morphological features as the dependent variables and group status (patients with a history of suicide attempt, patient controls and healthy controls) as the independent variable. All models included age, sex and IQ as covariates. Models with cortical surface area and subcortical volumes also included intracranial volume as a covariate. We used a $5 \%$ false discovery rate (FDR $)^{42}$ to correct for multiple comparisons; we applied this separately for each parameter tested (cortical thickness estimates, surface area estimates and subcortical volumes). The $p_{\mathrm{FDR}}$ values are reported as $q$ values. We calculated effect sizes for significant group differences using Hedges' $d$. Sensitivity analyses tested the effect of medication type (antidepressants, mood stabilizers, adjunct low-dose neuroleptics, benzodiazepines or stimulants). For these analyses, each medication class was coded as a dummy variable $(0=$ no medication, $1=$ medication) and included separately in the aforementioned linear regression models.

Next, to examine whether morphometric features (cortical thickness, cortical surface area and subcortical volumes) were differentially related to age in each group, we conducted linear regression models using the interaction of age and group, controlling for sex, IQ and intracranial volume (only for cortical surface areas and cortical thickness). We conducted additional sensitivity analyses to rule out the effect of psychotropic medication, in the same manner as for the basic linear models (see above). When the interaction of age and group was significant $(p \leq 0.05)$, we conducted post hoc group comparisons of the correlation coefficients between age and morphometric features (residualized for sex and IQ). We conducted these group comparisons with a bootstrapping procedure (5000 repetitions) that computed differences between correlation coefficients ( $r$ to $z$ converted with Fisher's transformation). Confidence intervals (95\%) that excluded 0 were deemed significant. ${ }^{43}$

\section{Results}

\section{Group demographic and clinical characteristics}

The demographic and clinical characteristics of the 3 groups of adolescents are presented in Table 1 . Female sex was predominant, but not different across groups $\left(\chi^{2}=1.4, p=0.5\right)$. The mean ( \pm standard deviation $[S D])$ age of all participants was $15.9 \pm 1.3$ years (range 11.6-18.1). Analysis of variance revealed a significant age difference between groups $\left(F_{2,89}=3.3\right.$, $p=0.04)$, but the post hoc Tukey test did not show any difference when groups were compared directly. Race/ethnicity was similar across groups. Level of parental education (our proxy measure of socioeconomic status ${ }^{44}$ ) indicated a higher proportion of parents whose highest educational achievement was elementary school in patients with a history of suicide attempt than in healthy controls. Diagnoses of depressive disorders differed slightly between patient groups: we found a higher rate of depressive disorder not otherwise specified relative to MDD in patients with a history of suicide attempt than in patient controls. Although the 2 patient groups did not differ in rates of attention-deficit/hyperactivity disorder diagnosis, stimulant use was more prevalent in patients with a history of suicide attempt than in patient controls. As expected, the 2 patient groups had levels of depressive symptoms that were similar, but higher than those of the healthy control group. Finally, among patients with a history of suicide attempt, the average (mean \pm SD) time between the last suicide attempt and the scanning session was $12.5 \pm$ 10.8 months (range $0.8-42.3$ ). The average number of suicide attempts was $2.0 \pm 1.6$ (range $1-7$ ) and the average age at first suicide attempt was $14.4 \pm 1.3$ years (range 10.6-16.6).

\section{Group differences in brain morphometry}

Analyses of covariance yielded significant group differences in surface area $(q<0.05)$ in 9 prefrontal regions (bilateral medial orbitofrontal cortex, right lateral orbitofrontal cortex, bilateral rostral middle frontal, left pars triangularis, right superior frontal, right precentral, left insula), 5 temporal regions (bilateral middle temporal, bilateral superior temporal, right fusiform) and 3 parietal regions (bilateral precuneus, right supramarginal; Figure 1 left and Appendix 1, Table S1, available at jpn.ca/200198-a1). Post hoc group comparisons indicated smaller surface area in patients with a history of suicide attempt than in healthy controls in all 17 regions, but no significant differences between patients with a history of suicide attempt and patient controls. Five regions that differed between patients with a history of suicide attempt and healthy controls did not differ significantly between patient controls and healthy controls: the left medial orbitofrontal cortex, the right lateral orbitofrontal cortex, the right rostral middle frontal cortex, the right superior frontal cortex and the right precentral cortex. No region was uniquely different between patient controls and healthy controls. The largest effect sizes between patients with a history of suicide attempt and healthy controls were in the right medial and lateral orbitofrontal cortex $(g=$ 0.82 and 0.66 , respectively) and the bilateral precuneus (left $g=$ 


\begin{tabular}{|c|c|c|c|c|c|c|}
\hline \multirow[b]{2}{*}{ Characteristic } & \multicolumn{3}{|c|}{ Group* $†$} & \multicolumn{3}{|c|}{ Group comparison } \\
\hline & $\begin{array}{l}\text { Patients with a history } \\
\text { of suicide attempt } \\
\qquad(n=28)\end{array}$ & $\begin{array}{l}\text { Patient controls } \\
\qquad(n=34)\end{array}$ & $\begin{array}{l}\text { Healthy } \\
\text { controls } \\
(n=30)\end{array}$ & $\chi^{2} / F$ & $p$ value & Post hoc \\
\hline Female & $24(86)$ & $27(79)$ & $22(73)$ & $\chi_{2}^{2}=1.4$ & 0.5 & - \\
\hline Age, yr & $16.2 \pm 1.0$ & $16.1 \pm 1.5$ & $15.4 \pm 1.3$ & $F_{2,89}=3.3$ & 0.04 & - \\
\hline \multicolumn{7}{|l|}{ Race/ethnicity } \\
\hline Asian & $<5$ & $<5$ & $<5$ & $\chi_{2}^{2}=0.3$ & 0.9 & \\
\hline Black & $<5$ & 0 & $<5$ & $\chi_{2}^{2}=5.8$ & 0.06 & \\
\hline White & $21(75)$ & $26(76)$ & $21(70)$ & $\chi_{2}^{2}=0.4$ & 0.8 & \\
\hline Indigenous & $<5$ & $6(18)$ & $<5$ & $\chi_{2}^{2}=4.0$ & 0.1 & \\
\hline Latin & 0 & $<5$ & $<5$ & $\chi_{2}^{2}=2.4$ & 0.3 & \\
\hline Multiethnic & $<5$ & $<5$ & 0 & $\chi_{2}^{2}=2.0$ & 0.4 & \\
\hline \multicolumn{7}{|l|}{ Parental education $\ddagger$} \\
\hline Elementary school & $7(25)$ & $3(9)$ & $0(0)$ & $\chi^{2}{ }_{2}=12.3$ & 0.002 & $\begin{array}{l}\text { Patients with a history of } \\
\text { suicide attempt }>\text { patient } \\
\text { controls, healthy controls }\end{array}$ \\
\hline High school & $5(18)$ & $6(18)$ & $6(20)$ & $\chi_{2}^{2}=0.1$ & 1.0 & \\
\hline College & $4(14)$ & $3(9)$ & $6(20)$ & $\chi_{2}^{2}=2.5$ & 0.3 & \\
\hline University & $12(43)$ & $22(65)$ & $17(57)$ & $\chi_{2}^{2}=2.7$ & 0.3 & \\
\hline IQ score§ & $102.3 \pm 15.9$ & $108.4 \pm 13.7$ & $110.5 \pm 13.0$ & $F_{2,91}=2.3$ & 0.1 & - \\
\hline Beck Depression Scale II score & $30.1 \pm 12.9$ & $24.8 \pm 12.5$ & $6.0 \pm 6.0$ & $F_{2,91}=39.5$ & $<0.001$ & $\begin{array}{c}\text { Patients with a history of } \\
\text { suicide attempt, patient } \\
\text { controls }>\text { healthy } \\
\text { controls }\end{array}$ \\
\hline \multicolumn{7}{|l|}{ Psychiatric diagnosis/history } \\
\hline Major depressive disorder & $14(50)$ & $25(74)$ & - & $\chi^{2}=3.6$ & 0.06 & - \\
\hline Dysthymia & $5(18)$ & $7(21)$ & - & $\chi^{2}=0.1$ & 0.8 & - \\
\hline $\begin{array}{l}\text { Depressive disorder not } \\
\text { otherwise specified }\end{array}$ & $10(36)$ & $4(12)$ & - & $\chi^{2}{ }_{1}=5.0$ & 0.03 & $\begin{array}{c}\text { Patients with a history of } \\
\text { suicide attempt > patient } \\
\text { controls }\end{array}$ \\
\hline $\begin{array}{l}\text { Anxiety disorder/post-traumatic } \\
\text { stress disorder }\end{array}$ & $12(43)$ & $17(50)$ & - & $\chi^{2}{ }_{1}=0.6$ & 0.43 & - \\
\hline Eating disorder & $<5$ & $<5$ & - & $\chi^{2}=0.1$ & 0.8 & - \\
\hline $\begin{array}{l}\text { Attention-deficit/hyperactivity } \\
\text { disorder }\end{array}$ & $9(32)$ & $4(12)$ & - & $\chi^{2}{ }_{1}=1.8$ & 0.2 & - \\
\hline $\begin{array}{l}\text { Nonsuicidal self-injury, lifetime } \\
\text { history }\end{array}$ & $24(86)$ & $22(65)$ & - & $\chi^{2}{ }_{1}=0.5$ & 0.5 & \\
\hline \multicolumn{7}{|l|}{ Psychotropic medication } \\
\hline Antidepressant & $14(50)$ & $21(62)$ & - & $\chi^{2}=0.4$ & 0.5 & - \\
\hline Mood stabilizer & $<5$ & $<5$ & - & $\chi^{2}{ }_{1}=0.02$ & 0.9 & - \\
\hline Low-dose neuroleptic & $11(39)$ & $9(26)$ & - & $\chi^{2}=1.2$ & 0.3 & - \\
\hline Benzodiazepine & $<5$ & $<5$ & - & $\chi^{2}{ }_{1}=0.02$ & 0.9 & - \\
\hline Stimulant & $8(29)$ & $<5$ & - & $\chi^{2}=5.8$ & 0.02 & $\begin{array}{c}\text { Patients with a history of } \\
\text { suicide attempt > patient } \\
\text { controls }\end{array}$ \\
\hline \multicolumn{7}{|c|}{$\begin{array}{l}\text { Data are presented as } n(\%) \text { or mean } \pm \text { standard deviation. Findings were significant at } p<0.05 \text {. } \\
\text { *Patients with a history of suicide attempt had a depressive disorder and a history of suicide attempt; patient controls had a depressive disorder but no history of suicide attempt; healthy } \\
\text { controls had no had no psychiatric disorder and no history of suicide attempt. } \\
\text { †Populations of fewer than } 5 \text { have been rounded to protect patient privacy. } \\
\text { †One value was missing for a healthy control case. } \\
\text { §Wechsler Intelligence Scale for Children or Wechsler Adult Intelligence Scale. }\end{array}$} \\
\hline
\end{tabular}

0.64 , right $g=0.64)$. Group differences in regional surface area remained significant when controlling for medication status.

The 3 groups did not differ significantly in terms of regional cortical thickness (Appendix 1, Table S2).

The 3 groups did differ significantly in subcortical volumes of the left amygdala $\left(F_{2,85}=7.66, q=0.009\right)$, right thalamus $\left(F_{2,85}=6.28, q=0.013\right)$ and right nucleus accumbens $\left(F_{2,85}=7.15\right.$, $q=0.009 ;$ Figure 1 right, Appendix 1, Table S3). Post hoc group comparisons indicated smaller volumes in all 3 regions in patients with a history of suicide attempt compared to healthy controls, but not patient controls. The largest effect size was found in the left amygdala $(g=0.74)$. Group differences in subcortical volumes remained significant when controlling for medication status. 


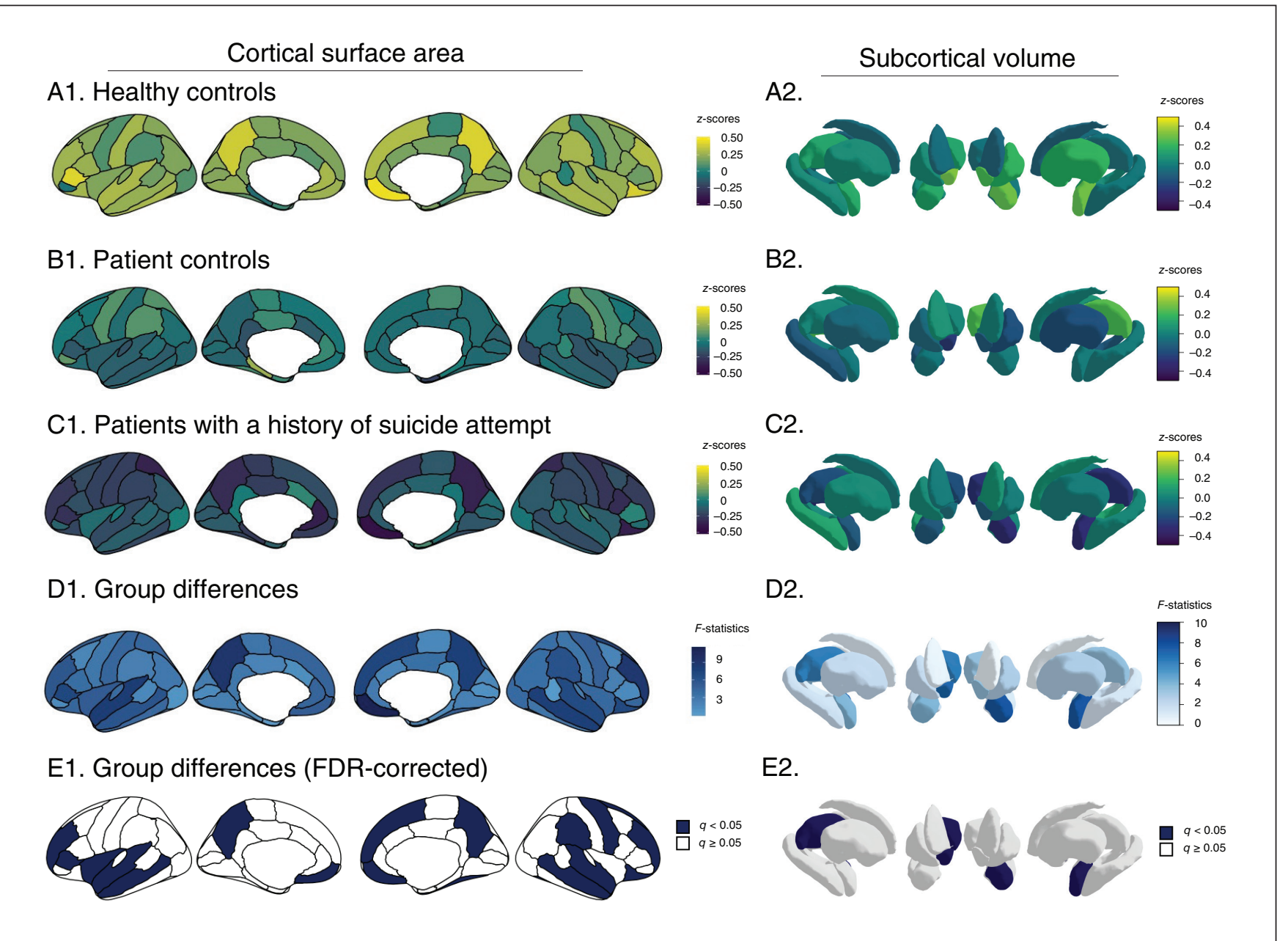

Fig. 1: Group differences in cortical surface area (left) and subcortical volume (right). A-C: within-group analyses; D-E: betweengroup comparisons. FDR = false discovery rate.

The effects of the covariates (sex, age, IQ, intracranial volume) for each structural feature are reported in Appendix 1, Tables S5 to S7.

\section{Age $\times$ group interaction analyses}

To explore the possibility that brain morphologic features mature differently as a function of age and history of suicide attempt, we examined the interactions of age and group.

We found no significant interaction of age and group for cortical surface areas. In contrast, we did find significant interactions of age and group for cortical thickness in the left superior temporal cortex $\left(F_{2,84}=3.8, p=0.03\right)$, right inferior temporal cortex $\left(F_{2,84}=4.4, p=0.02\right)$ and right insula $\left(F_{2,84}=\right.$ $4.4, p=0.02$; Figure 2 left and Appendix 1, Table S4). Post hoc analyses indicated significant differences in these regions between patients with a history of suicide attempt and both patient controls and healthy controls: whereas age was positively correlated with cortical thickness (adjusted for sex and IQ) in patients with a history of suicide attempt, correlations were negative in patient controls and healthy controls.
For subcortical regions, we found a significant interaction of age and group for the volumes of the right putamen $\left(F_{2,84}=7.6, p=0.007\right)$ and left hippocampus $\left(F_{2,84}=4.7, p=\right.$ 0.03; Figure 2 right and Appendix 1, Table S4). Post hoc analyses yielded a significant difference only in the right putamen between patients with a history of suicide attempt and both patient controls and healthy controls; age and volume (adjusted for sex and IQ) were correlated positively in patients with a history of suicide attempt and negatively in patient controls and healthy controls.

Controlling for medication status did not affect the significance of age $\times$ group interactions for regional cortical thickness and subcortical volumes.

\section{Discussion}

The first objective of the current study was to examine whether a history of suicide attempt in adolescents with depression was associated with alterations in brain morphology (including cortical surface area and thickness) and subcortical volumes. We found smaller surface areas in 
Cortical thickness $\times$ age

\section{A1. Healthy controls}
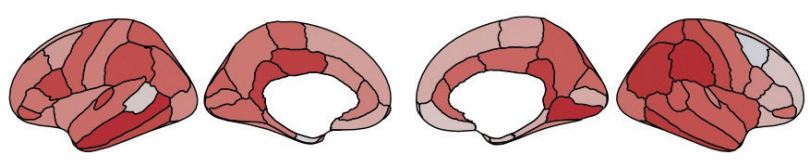

B1. Patient controls
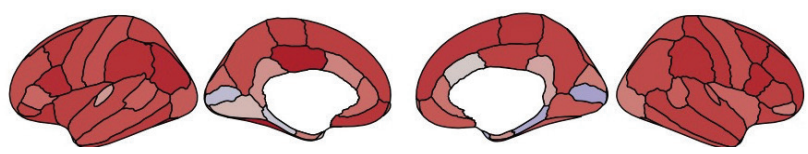

C1. Patients with a history of suicide attempt
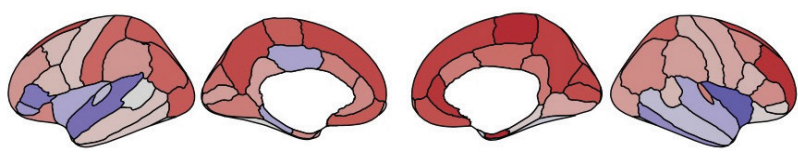

D1. Group differences
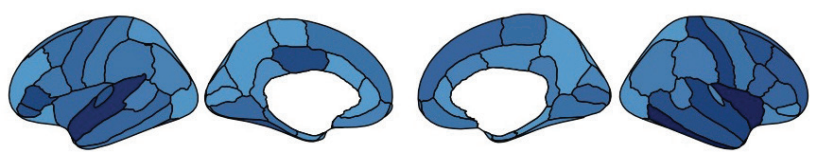

E1. Group differences
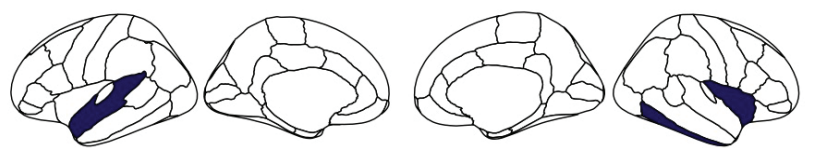

\section{Subcortical volume $\times$ age}

A2.

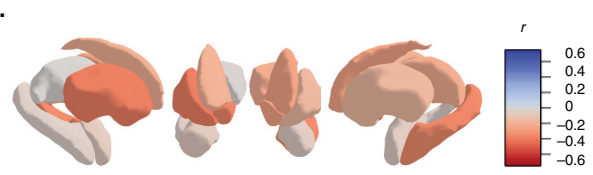

B2.

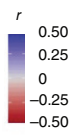

C2.
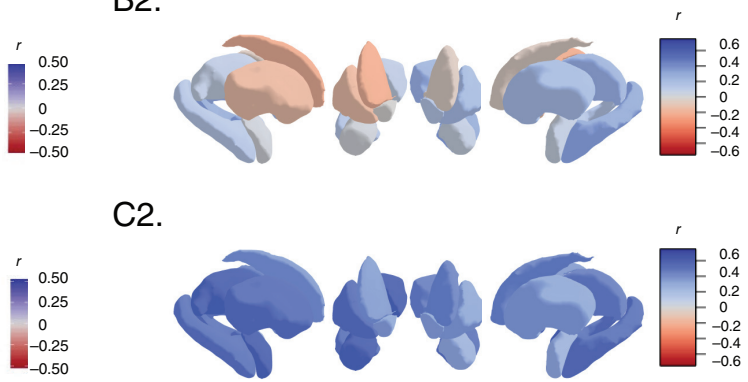

D2.

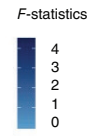

E2.
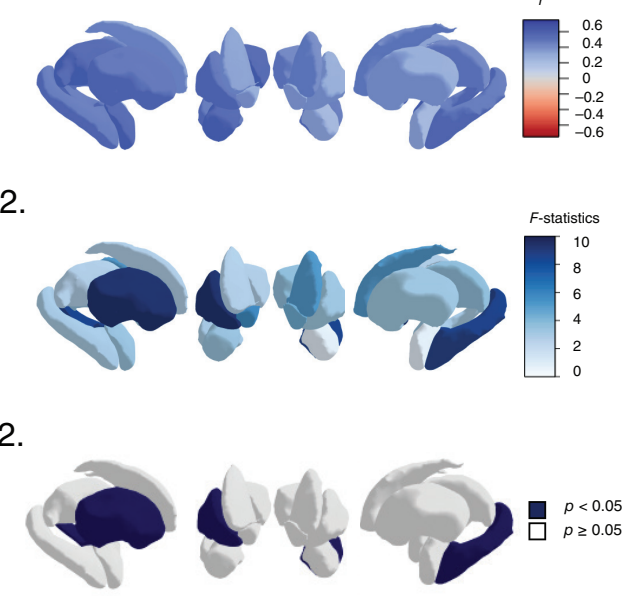

Fig. 2: Regional correlations between age and cortical thickness and group comparisons for the interaction between age and subcortical volumes. A-C: within-group analyses; D-E: between-group comparisons.

9 prefrontal regions, 5 temporal regions and 3 parietal regions in patients with a history of suicide attempt than in healthy controls, but these cortical alterations did not distinguish patients with a history of suicide attempt from patient controls. Similarly, we found smaller subcortical volumes in the left amygdala, right thalamus and right nucleus accumbens in patients with a history of suicide attempt than in healthy controls, but not patient controls. Our findings also indicated that cortical thickness was not different in patients with a history of suicide attempt compared to patient controls or healthy controls. Thus, our results suggest that patients with a history of suicide attempt show subtle alterations in morphological measurements in a widespread network of cortical and subcortical regions, but that these changes do not distinguish patients with a history of suicide attempt from patient controls.

Several of our findings in cortical regions were consistent with the results of previous studies. Specifically, we replicated findings in the right superior temporal gyrus that were reported in 2 independent studies that also found smaller volumes in adolescents with depression and a history of suicide attempt ${ }^{17,18}$ than in healthy controls. In addition, volu- metric anomalies in the orbitofrontal cortex were reported compared to healthy controls in young patients with bipolar disorder and a history of suicide attempt. ${ }^{20}$ Our study suggests that alterations in surface area, rather than cortical thickness, explain these previously observed volumetric differences associated with youth suicidal behaviours. With respect to anomalies in subcortical structure, previous reports in adolescent suicide behaviours are rare. Nevertheless, our findings were consistent with converging evidence implicating alterations in limbic subcortical structures in adolescent depressive disorders ${ }^{45}$ but also confirmed that these anomalies were not reliable markers of suicide history, parallelling reports in adult samples. ${ }^{46,47}$ Although structural markers may not enhance diagnostic capacities, our findings support the view that neurobiological alterations contribute to the suicidal diathesis. Additional neurobiological factors - at the functional, neuronal, hormonal or molecular levels and environmental differences are probably involved in the specific mechanisms involved in suicidal behaviours. ${ }^{7,48}$

Although group comparisons did not yield robust differences, our analyses also suggested that the relationship between age and morphological measures is different in patients 
with a history of suicide attempt compared to both control groups. These results should be interpreted with caution given their exploratory nature, but they highlight a possible link between atypical brain development and the emergence of suicide risk in adolescents with depressive disorders. More specifically, we found localized abnormalities in the relationship between chronological age and cortical thickness in the right insula and temporal regions. Although average cortical thickness in those regions was negatively associated with age in the patient control and healthy control groups (which is normative in adolescents) ${ }^{49,50}$ cortical thickness in these same regions was positively associated with age in patients with a history of suicide attempt. It should be noted that patients with a history of suicide attempt and patient controls also differed meaningfully in their clinical profile (a larger number of patients with depressive disorder not otherwise specified in the group with a history of suicide attempt) and parental background (lower levels of parental education among patients with a history of suicide attempt). Clinical and environmental heterogeneity might at least partially explain some of the observed effects, which could be parsed with longitudinal studies in larger samples.

The insula has been associated with suicidality in adolescents with depression. A recent study found that the insula was among several regions with increased activity elicited by implicit self-associations with death. ${ }^{51}$ Schwartz and colleagues ${ }^{52}$ found that longitudinal reductions in the coherence of the salience network - which is typically anchored in the insula ${ }^{53}$ - was associated with change between the worst severity of suicidal ideation and current levels of suicidal ideation. Collectively, these results suggest that agerelated alterations in the insula are associated with adolescent suicidal risk, particularly in relation to emotionally salient or self-referential contexts.

Our exploratory analysis also suggests that age-related anomalies in temporal thickness are associated with adolescent suicidal behaviour. Interestingly, the temporal cortex is the region with the most protracted grey matter change in adolescence; some changes in cortical thickness continue well into young adulthood. ${ }^{25,50}$ The temporal cortex — particularly the superior temporal gyrus - is a highly associative region that has been implicated in high-order semantic processing. ${ }^{54}$ Age-related anomalies in thickness of the temporal lobes have been linked with autism, ${ }^{55}$ a neurodevelopmental disorder characterized by mentalization deficits. Hence, the possible link between atypical adolescent maturation in the temporal cortex and suicidal behaviour might be related to cognitive processes involved in self-regulation or mentalization.

We also found an age-related association with grey matter volume in the right putamen. Again, patients with a history of suicide attempt were characterized by a positive correlation between age and putamen volume, and the healthy controls and patient controls were characterized by negative correlations, consistent with normative developmental patterns of the putamen during this stage..$^{56}$ The putamen plays an important role in value-based decision-making and learning in goal-directed behaviours. ${ }^{57}$ Thus, abnormal maturation of the putamen may be linked to impaired decision-making or to other reward-based processes in adolescent suicidal behaviours. It is unclear whether maturational changes in subcortical regions are linked to abnormal function, as has been observed at later stages of the lifespan. ${ }^{58}$

Although our results could not be used to elucidate the mechanisms that might underlie abnormal maturational patterns in specific brain regions in adolescents with a history of suicide attempt, several hypotheses can be generated for future investigation. Macroscopic alterations in grey matter have been linked to both microscopic changes in synaptic density (pruning) and white matter development, such as myelination. ${ }^{59,60}$ In key prefrontal and limbic regions involved in stress reactivity, emotion and reward regulation, several of these processes are highly sensitive to exposure to early-life stress, and even prenatal conditions. ${ }^{61}$ Early-life stress affects brain development through complex epigenetic mechanisms, such as altered methylation patterns, histone acetylation or expression of post-transcriptional regulators. ${ }^{16}$ In addition, stress-linked neuroinflammation and glial alterations might underlie the molecular mechanisms related to atypical brain development in adolescents. ${ }^{48}$

\section{Limitations}

We should underscore a few limitations of this study. First, although we recruited a large number of participants, we may not have had sufficient statistical power to detect effects that could survive a highly conservative level of correction. Second, the age range was relatively wide, which limited our capacity to fully account for developmental variability. Nevertheless, this age range still enabled the detection of age-related structural differences spanning adolescence. Third, clinical heterogeneities among our sample — including the characteristics of suicidal behaviours, psychiatric comorbidities, and familial and environmental factors - may have affected our results and prevent generalization to other clinical populations. It also remains possible that the patient control group, which was recruited in a highly specialized clinic, might have comprised adolescents who were at risk of suicide but had not yet made an attempt. This unavoidable misclassification probably reduced the differences between the 2 patient groups. On the technical side, although the automated procedure for subcortical segmentation we used has been deployed in pediatric populations, ${ }^{56}$ its subcortical template was derived from adult brains (age $>18$ years) ${ }^{37}$ Age-specific segmentation techniques might yield more accurate measurements of subcortical volumes in the developing brain. ${ }^{62}$ Furthermore, the results might have been constrained by the chosen parcellation (Desikan-Killiany atlas), which might have reduced our capacity to detect variations crossing predetermined anatomic boundaries. Using more fine-grained parcellations would require a larger sample. Additionally, recent data indicate that MRI head coils with less than 32 channels might yield a less homogeneous signal-to-noise ratio and alter tissue segmentation, suggesting that improvement in scanning hardware might provide more reliable structural findings. ${ }^{63}$ Finally, although controlling for medication status did not affect our findings, we did 
not account for more complex pharmacological effects, such as dose effects, duration of treatment and interactions. Future research with large-scale neuroimaging studies is required to disentangle the independent and mutual effect of biological and psychosocial factors in adolescent suicide risk.

This study adds to our understanding of the brain structural mechanisms that may be associated with suicidal behaviours in adolescence, but several additional steps are needed before structural neuroimaging is integrated into predictive algorithms for adolescent suicide risk. Indeed, our main finding precludes the use of brain morphometric features as discrete biomarkers of adolescent suicide risk. First, it is critical that future studies corroborate our age-related findings prospectively. Our results were generated at the group level, but clinical use of brain maturational indices will require quantifying deviations from normative developmental patterns. Second, approaching the brain as a complex network and combining neuroimaging modalities might provide a more sensitive approach for the detection of people at high risk. Finally, novel statistical methods using machine learning might assist in the integration of multimodal neuroimaging assessments and provide classification algorithms that are more accurate than traditional multivariate methods. ${ }^{64}$ Finally, studies recruiting across a broader psychiatric spectrum (including bipolar disorder, psychotic disorders and other neuropsychiatric disorders) are needed to elucidate whether risk-related neural markers are transdiagnostic or disorder-specific biosignatures.

\section{Conclusion}

Our study suggests that subtle morphological alterations in surface area in the prefrontal lobes and in the parietal and temporal lobes, as well as in the volume of several subcortical nuclei, are associated with a history of suicidal behaviours in adolescents with depressive disorders. However, these alterations may not be sufficient to identify patients at greatest risk for suicidal behaviours in a group diagnosed with a depressive disorder. Our findings also indicate atypical agerelated correlations among patients with a history of suicide attempt relative to other groups in regions involved in saliency processing, higher-order cognition and rewardoriented behaviour, which might contribute to the risk of suicidal behaviours in adolescents with depression.

Acknowledgements: This research was financed by Manulife Research Fund in Teen Depression, which supports the Manulife Centre for Breakthroughs in Teen Depression and Suicide Prevention. AJG was supported the Fonds de Recherche du Québec-Santé (FRQS/MSSS Resident Physician Health Research Career Training Program). MMC was supported by a Junior 2 Research Scholar Salary from the FRQS. ML was supported by a Research Chair from FRQS and from a James McGill Professorship. MCG holds a Canada Research Chair Tier-2 and a Young Investigator Award of the American Foundation for Suicide Prevention. IHG was supported by the National Institutes of Health (NIH; R37MH101495) and the Stanford University Precision Health and Integrated Diagnostics Center. TCH was supported by the National Institutes of Health (NIH; K01MH117442), the Stanford University Precision Health and Integrated Diagnostics Center and The Ray and Dagmar Dolby Family Fund. The content is solely the responsibility of the authors. The funding agencies played no role in the design and conduct of the study; collection, management, analysis, and interpretation of the data; and preparation, review, or approval of the manuscript. We thank Daysi Zentner, Geneviève Laurent, and Léa Perret for their assistance with data collection and organization. Finally, we thank the participants and their families participating in this study as well as the clinicians involved with adolescents and their families (Theodora Mikedis, Jean-Chrysostome Zanga, Didier Blondin-Lavoie).

Affiliations: From the McGill Group for Suicide Studies, Douglas Mental Health University Institute, Montréal, Canada (Gifuni, Geoffroy, Turecki, Renaud, Jollant); the Department of Psychiatry, McGill University, Montréal, Canada (Gifuni, Chakravarty, Lepage, Turecki, Renaud, Jollant); the Manulife Centre for Breakthroughs in Teen Depression and Suicide Prevention, Montréal, Canada (Gifuni, Renaud); the Department of Psychology, Stanford University, Stanford, USA (Gifuni, Gotlib); the Department of Psychiatry \& Behavioral Sciences and Weill Institute for Neurosciences, University of California at San Francisco, San Francisco, USA (Ho); the Department of Educational and Counselling Psychology, McGill University, Montréal, Canada (Geoffroy); the Department of Sociology, Université de Montréal, Montréal, Canada (Lacourse); the Université de Paris, Paris, France (Jollant); the GHU Paris Psychiatrie et Neurosciences, Hôpital Sainte-Anne, Paris, France (Jollant); and the Academic Hospital (CHU) Nîmes, Nîmes, France (Jollant).

Competing interests: M. Chakravarty is an associate editor of JPN; he was not involved in the review or decision to accept this manuscript for publication. M. Lepage reports grants from Otsuka Lundbeck Alliance, diaMentis and Roche, research support from Cogsate and personal fees from Otsuka, all outside the submitted work. No other competing interests declared.

Contributors: M.-C. Geoffroy, E. Lacourse, J. Renaud and F. Jollant designed the study. A. Gifuni, J. Renaud and F. Jollant acquired the data, which A. Gifuni, M. Chakravarty, M. Lepage, T. Ho, E. Lacourse, I. Gotlib, G. Turecki, J. Renaud and F. Jollant analyzed. A. Gifuni wrote the article, which M. Chakravarty, M. Lepage, T. Ho, M.-C. Geoffroy, E. Lacourse, I. Gotlib, G. Turecki, J. Renaud and F. Jollant reviewed. All authors approved the final version to be published and can certify that no other individuals not listed as authors have made substantial contributions to the paper.

Content licence: This is an Open Access article distributed in accordance with the terms of the Creative Commons Attribution (CC BY-NC-ND 4.0) licence, which permits use, distribution and reproduction in any medium, provided that the original publication is properly cited, the use is noncommercial (i.e., research or educational use), and no modifications or adaptations are made. See: https://creativecommons.org/licenses/by-nc-nd/4.0/

\section{References}

1. Mokdad AH, Forouzanfar MH, Daoud F, et al. Global burden of diseases, injuries, and risk factors for young people's health during 1990-2013: a systematic analysis for the Global Burden of Disease Study 2013. Lancet 2016;387:2383-401.

2. Mortier P, Auerbach RP, Alonso J, et al. Suicidal thoughts and behaviors among first-year college students: results from the WMH-ICS Project. J Am Acad Child Adolesc Psychiatry 2018;57:263273.e1.

3. Nock MK, Green JG, Hwang I, et al. Prevalence, correlates, and treatment of lifetime suicidal behavior among adolescents: results from the National Comorbidity Survey Replication Adolescent Supplement. JAMA Psychiatry 2013;70:300-10.

4. Reinherz HZ, Tanner JL, Berger SR, et al. Adolescent suicidal ideation as predictive of psychopathology, suicidal behavior, and compromised functioning at age 30. Am J Psychiatry 2006;163:1226-32.

5. Kleiman EM, Turner BJ, Fedor S, et al. Examination of real-time fluctuations in suicidal ideation and its risk factors: results from two ecological momentary assessment studies. J Abnorm Psychol 2017;126:726-38.

6. Franklin JC, Ribeiro JD, Fox KR, et al. Risk factors for suicidal thoughts and behaviors: a meta-analysis of 50 years of research. Psychol Bull 2017;143:187-232. 
7. Mann JJ, Rizk MM. A brain-centric model of suicidal behavior. Am J Psychiatry 2020;177:902-16.

8. Auerbach RP, Pagliaccio D, Allison GO, et al. Neural correlates associated with suicide and non-suicidal self-injury in youth. Biol Psychiatry 2020;89:119-133.

9. Schmaal L, van Harmelen A-L, Chatzi V, et al. Imaging suicidal thoughts and behaviors: a comprehensive review of 2 decades of neuroimaging studies. Mol Psychiatry 2020;25:408-27.

10. Geschwind DH, Rakic P. Cortical revolution: judge the brain by its cover. Neuron 2013;80:633-47.

11. Kremen WS, Fennema-Notestine C, Eyler LT, et al. Genetics of brain structure: contributions from the Vietnam Era Twin Study of Aging. Am J Med Genet B Neuropsychiatr Genet 2013;162B:751-61.

12. Chen C-H, Fiecas M, Gutiérrez ED, et al. Genetic topography of brain morphology. Proc Natl Acad Sci U S A 2013;110:17089-94.

13. Campos AI, Thompson PM, Veltman DJ, et al. Brain correlates of suicide attempt in 18,925 participants across 18 international cohorts. medRxiv;2020: doi:10.1101/2020.05.06.20090191

14. Wolff M, Vann SD. The cognitive thalamus as a gateway to mental representations. J Neurosci 2019;39:3-14.

15. Napier TC, Mickiewicz AL. The role of the ventral pallidum in psychiatric disorders. Neuropsychopharmacology 2010;35:337-337.

16. Turecki G, Ernst C, Jollant F, et al. The neurodevelopmental origins of suicidal behavior. Trends Neurosci 2012;35:14-23.

17. Pan LA, Ramos L, Segreti A, et al. Right superior temporal gyrus volume in adolescents with a history of suicide attempt. Br J Psychiatry 2015;206:339-40.

18. McLellan Q, Wilkes TC, Swansburg R, et al. History of suicide attempt and right superior temporal gyrus volume in youth with treatmentresistant major depressive disorder. J Affect Disord 2018;239:291-4.

19. Fan S, Lippard ETC, Sankar A, et al. Grey and white matter differences in adolescents and young adults with prior suicide attempts across bipolar and major depressive disorders. J Affect Disord 2019; 245:1089-97.

20. Johnston JAY, Wang F, Liu J, et al. Multimodal neuroimaging of fronto-limbic structure and function associated with suicide attempts in adolescents and young adults with bipolar disorder. Am J Psychiatry 2017; 174:667-75.

21. Lippard ETC, Johnston JAY, Spencer L, et al. Preliminary examination of gray and white matter structure and longitudinal structural changes in frontal systems associated with future suicide attempts in adolescents and young adults with mood disorders. J Affect Disord 2019;245:1139-48.

22. Gifuni AJ, Perret LC, Lacourse E, et al. Decision-making and cognitive control in adolescent suicidal behaviors: a qualitative systematic review of the literature. Eur Child Adolesc Psychiatry 2020 [Epub ahead of print]. doi: 10.1007/s00787-020-01550-3.

23. Ho TC, Cichocki AC, Gifuni AJ, et al. Reduced dorsal striatal gray matter volume predicts implicit suicidal ideation in adolescents. Soc Cogn Affect Neurosci 2018;13:1215-24.

24. Casey BJ. Beyond simple models of self-control to circuit-based accounts of adolescent behavior. Annu Rev Psychol 2015;66:295-319.

25. Tamnes CK, Herting MM, Goddings A-L, et al. Development of the cerebral cortex across adolescence: a multisample study of inter-related longitudinal changes in cortical volume, surface area, and thickness. J Neurosci 2017;37:3402-12.

26. Vijayakumar N, Allen NB, Youssef G, et al. Brain development during adolescence: a mixed-longitudinal investigation of cortical thickness, surface area, and volume. Hum Brain Mapp 2016;37:2027-38.

27. Fish AM, Nadig A, Seidlitz J, et al. Sex-biased trajectories of amygdalo-hippocampal morphology change over human development. Neuroimage 2020;204:116122.

28. Brent DA, Mann JJ. Family genetic studies, suicide, and suicidal behavior. Am J Med Genet C Semin Med Genet 2005;133C:13-24.

29. Jollant F, Wagner G, Richard-Devantoy S, et al. Neuroimaginginformed phenotypes of suicidal behavior: a family history of suicide and the use of a violent suicidal means. Transl Psychiatry 2018;120:1-10

30. Posner K, Oquendo MA, Gould M, et al. Columbia Classification Algorithm of Suicide Assessment (C-CASA): classification of sui- cidal events in the FDA's pediatric suicidal risk analysis of antidepressants. Am J Psychiatry 2007;164:1035-43.

31. Silverman MM, Berman AL, Sanddal ND, et al. Rebuilding the Tower of Babel: a revised nomenclature for the study of suicide and suicidal behaviors part 2: suicide-related ideations, communications, and behaviors. Suicide Life Threat Behav 2007;37:264-77.

32. Kaufman J, Birmaher B, Brent DA, et al. K-SADS-PL. I Am Acad Child Adolesc Psychiatry 2000;39:1208.

33. Kaufman J, Birmaher B, Brent D, et al. Schedule for Affective Disorders and Schizophrenia for School-Age Children-Present and Lifetime version (K-SADS-PL): initial reliability and validity data. J Am Acad Child Adolesc Psychiatry 1997;36:980-8.

34. Beck AT, Steer RA, Brown G. Manual for the Beck Depression Inventory II. San Antonio (TX): Psychological Corporation; 1996.

35. Wechsler D. Wechsler Intelligence Scale for Children. 4th ed. San Antonio (TX): Psychological Corporation; 2003.

36. Wechsler D. Wechsler Adult Intelligence Scale. 4th ed. Bloomington (MN): Pearson Assessment; 2008.

37. Fischl B, Salat DH, Busa E, et al. Whole brain segmentation: automated labeling of neuroanatomical structures in the human brain. Neuron 2002;33:341-55.

38. Fischl B, Dale AM. Measuring the thickness of the human cerebral cortex from magnetic resonance images. Proc Natl Acad Sci U S A 2000;97:11050-5.

39. Desikan RS, Ségonne F, Fischl B, et al. An automated labeling system for subdividing the human cerebral cortex on MRI scans into gyral based regions of interest. Neuroimage 2006;31:968-80.

40. R Core Team. R: A language and environment for statistical computing. Vienna: R Foundation for Statistical Computing; 2019. Available: www.R-project.org (accessed 2021 Apr. 21).

41. RStudio Team. RStudio: integrated development environment for $R$. Boston (MA): RStudio, Inc.; 2016. Available: www.rstudio.com (accessed 2021 Apr. 21).

42. Benjamini $\mathrm{Y}$, Hochberg $\mathrm{Y}$. Controlling the false discovery rate: a practical and powerful approach to multiple testing. $J R$ Stat Soc B 1995;57:289-300.

43. Zou GY. Toward using confidence intervals to compare correlations. Psychol Methods 2007;12:399-413.

44. Svedberg P, Nygren JM, Staland-Nyman C, et al. The validity of socioeconomic status measures among adolescents based on selfreported information about parents occupations, FAS and perceived SES; implication for health related quality of life studies. BMC Med Res Methodol 2016;16:48.

45. Schmaal L, Hibar DP, Sämann PG, et al. Cortical abnormalities in adults and adolescents with major depression based on brain scans from 20 cohorts worldwide in the ENIGMA Major Depressive Disorder Working Group. Mol Psychiatry 2017;22:900-9.

46. Gifuni AJ, Ding Y, Olié E, et al. Subcortical nuclei volumes in suicidal behavior: nucleus accumbens may modulate the lethality of acts. Brain Imaging Behav 2016;10:96-104.

47. Rentería ME, Schmaal L, Hibar DP, et al. Subcortical brain structure and suicidal behaviour in major depressive disorder: a metaanalysis from the ENIGMA-MDD working group. Transl Psychiatry 2017;7:e1116

48. Lutz P-E, Mechawar N, Turecki G. Neuropathology of suicide: recent findings and future directions. Mol Psychiatry 2017;22: 1395-412.

49. Ducharme S, Albaugh MD, Nguyen T-V, et al. Trajectories of cortical thickness maturation in normal brain development- the importance of quality control procedures. Neuroimage 2016;125:267-79.

50. Gogtay N, Giedd JN, Lusk L, et al. Dynamic mapping of human cortical development during childhood through early adulthood. Proc Natl Acad Sci U S A 2004;101:8174-9.

51. Ballard ED, Reed JL, Szczepanik J, et al. Functional imaging of the implicit association of the self with life and death. Suicide Life Threat Behav 2019;49:1600-8.

52. Schwartz J, Ordaz SJ, Ho TC, et al. Longitudinal decreases in suicidal ideation are associated with increases in salience network coherence in depressed adolescents. I Affect Disord 2019, 245:545-52. 
53. Uddin LQ. Salience processing and insular cortical function and dysfunction. Nat Rev Neurosci 2015;16:55-61.

54. Hein G, Knight RT. Superior temporal sulcus — it's my area: or is it? J Cogn Neurosci 2008;20:2125-36.

55. Wallace GL, Dankner N, Kenworthy L, et al. Age-related temporal and parietal cortical thinning in autism spectrum disorders. Brain 2010;133:3745-54.

56. Wierenga L, Langen M, Ambrosino S, et al. Typical development of basal ganglia, hippocampus, amygdala and cerebellum from age 7 to 24 . Neuroimage 2014;96:67-72.

57. Haber SN. Corticostriatal circuitry. Dialogues Clin Neurosci 2016;18:7-21.

58. Dombrovski AY, Siegle GJ, Szanto K, et al. The temptation of suicide: striatal gray matter, discounting of delayed rewards, and suicide attempts in late-life depression. Psychol Med 2012;42:1203-15.

59. Benes FM, Turtle M, Khan Y, et al. Myelination of a key relay zone in the hippocampal formation occurs in the human brain during childhood, adolescence, and adulthood. Arch Gen Psychiatry 1994;51:477-84.

60. Huttenlocher PR, Dabholkar AS. Regional differences in synaptogenesis in human cerebral cortex. J Comp Neurol 1997;387: 167-78.

61. O'Donnell KJ, Meaney MJ. Fetal origins of mental health: the developmental origins of health and disease hypothesis. Am J Psychiatry 2016;174:319-28.

62. Loh WY, Connelly A, Cheong JLY, et al. A new MRI-based pediatric subcortical segmentation technique (PSST). Neuroinformatics 2016;14:69-81.

63. Panman JL, To YY, van der Ende EL, et al. Bias introduced by multiple head coils in mri research: an 8 channel and 32 channel coil comparison. Front Neurosci 2019;13:729.

64. Belsher BE, Smolenski DJ, Pruitt LD, et al. Prediction models for suicide attempts and deaths: a systematic review and simulation. JAMA Psychiatry 2019;76:642-51. 